\title{
Designing a socially integrated mobile robot for ethological research ${ }^{\text {th }}$
}

\author{
A. Gribovskiya,**, J. Halloy ${ }^{\mathrm{b}, *}$, J.L. Deneubourg ${ }^{\mathrm{c}}$, F. Mondada ${ }^{\mathrm{a}}$ \\ ${ }^{a}$ Robotic Systems Laboratory 1, Ecole Polytechnique Fédérale de Lausanne, ME B3 30 \\ (Bâtiment ME), Station 9, CH-1015 Lausanne \\ ${ }^{b}$ Laboratoire Interdisciplinaire des Energies de Demain (LIED), Université Diderot Paris \\ VII, Batiment Condorcet, 10 Rue Alice Domon, 75205 Paris Cedex 13, France \\ ${ }^{c}$ Service d'Ecologie Sociale, Université libre de Bruxelles, ULB CP 231, Campus de la \\ Plaine, Bd du Triomphe, B-1050 Bruxelles, Belgique
}

\begin{abstract}
A robot introduced into an animal group, accepted by the animals as conspecifics, and capable of interacting with them is a very efficient tool for ethological research, particularly in studies of collective and social behavior. In this paper, we present the implementation of an autonomous mobile robot developed by the authors to study group behavior of chicks of the domestic chicken (Gallus gallus domesticus). We discuss the design of the robot and of the experimental setup that we built to run animal-robot experiments. The robot design was experimentally validated, it was demonstrated that the robot can be socially integrated into animal groups. The designed system opens new opportunities in the study of behavior in domestic fowl by using mobile robots. Being socially integrated into the animal group, mobile robots can profit from the positive feedback mechanism that plays key roles in animal collective behavior. They have potential applications in various domains, from pure scientific research to applied areas such as control and ensuring welfare of poultry.
\end{abstract}

Keywords: Animal-robot interaction, Autonomous mobile robots, Visual

\footnotetext{
The authors would like to thank the Swiss National Science Foundation for the support of this work and all the colleagues who contributed to this work.

${ }^{*}$ Corresponding author

** Principal corresponding author

Email addresses: alexey.gribovskiy@epfl.ch (A. Gribovskiy), jose.halloy@univ-paris-diderot.fr (J. Halloy), jldeneub@ulb.ac.be (J.L. Deneubourg), francesco.mondada@epfl.ch (F. Mondada)
}

Preprint submitted to Elsevier

February 22, 2018 
tracking, Sound localization, Collective animal behaviour

\section{Introduction}

Many animal species live in groups [1], group living gives to an individual many benefits such as reduced predation risk, better foraging efficiency and easier mate access. In exchange group living demands animals to synchronize their activities. Collective movement of insects, fish, birds or herds of ungulates is among the most remarkable examples of such synchronization [2]. It is commonly accepted that the collective movement phenomenon emerges as a result of interactions between individuals, but precise individual decision making mechanism and the role of the behavior of other group members in this mechanism is still a question under study [3].

In our research we use the domestic chicken (Gallus gallus domesticus) as a model of social bird species. We apply our study to small groups allowing the analysis of the behavior of all individuals with good precision. Under natural conditions, chickens live together in flocks forming social groups [4]. The key question we are interested in is how to establish the link between the individual behavior and the collective response (e.g., how the local interactions among group members maintain spatial cohesion). Most of the models of collective behavior hypothesize that individuals are similar and thus involve the same interaction rules. Whether this assumption holds in all cases is questionable. Many species are capable of inter-individual recognition and/or display stable relationships among group members. However, few studies have tackled collective decision as a function of social relationships and the role of a leader [5]. Our main objective is to study the link between individual behavior, interaction between individuals, and collective responses. To solve this question, the classical approach combines theoretical models and experiments at the individual and collective levels. In some studies animals trained to perform specific movements were introduced to the group to study this link $[6,7]$. The alternative is a recent approach to use robotic devices to study animal behavior [8]. The main idea is 
to make robots be accepted by animals as conspecifics or heterospecifics. Once this goal is achieved, the robot can be programmed to execute specific behaviors, and the animal response is observed. It makes this approach very efficient, particularly in studies of collective and social behavior, comparing with aforementioned methods based on pure behavioral observations or use of specifically trained animals. Indeed, robots allow us to test the animal response to various signals very precisely, as every element of the robot behavior, including the signals emitted, can be individually controlled.

In [9] specially designed autonomous mobile robots were introduced as conspecifics into groups of cockroaches. A mixed group were released on the arena with two shelters of different opacity, and a collective decision-making process of shelter selection were studied. It was demonstrated that robots can modulate the collective decision-making process and produce a global pattern not observed in their absence. These results demonstrate the possibility of using robots to study and control animal group behavior.

Robotic fishes presented in $[10,11,12]$ were designed to interact with live fish. In these studies robots share the same mechanical design approach, they consist of two modules: a replica fish fixed on the magnetic base and a miniature mobile robot guiding the replica fish from below the experimental tank.

Different robotic devices were also used to interact with flocks of birds. For instance, in [13] a remotely controlled robotic chick was used to study an aggregation behavior in Australian brush-turkey. An influence of the autonomous robot on the exploratory behavior in quail chicks were studied in [14]; for this the robot was equipped with a heat source stimulating chicks to follow it.

In this paper, we present the implementation of an autonomous mobile robot developed by the authors to study the group behavior of chicks. The robot was integrated into animal groups as a surrogate hen and was accepted by animals thanks to the filial imprinting mechanism. Three sample snapshots from experimental videos are presented on Figure 1, they show chicks interacting with one or several robots, solitary and in groups. In this study our main interest was the collective movement phenomenon and the role of the leader represented 

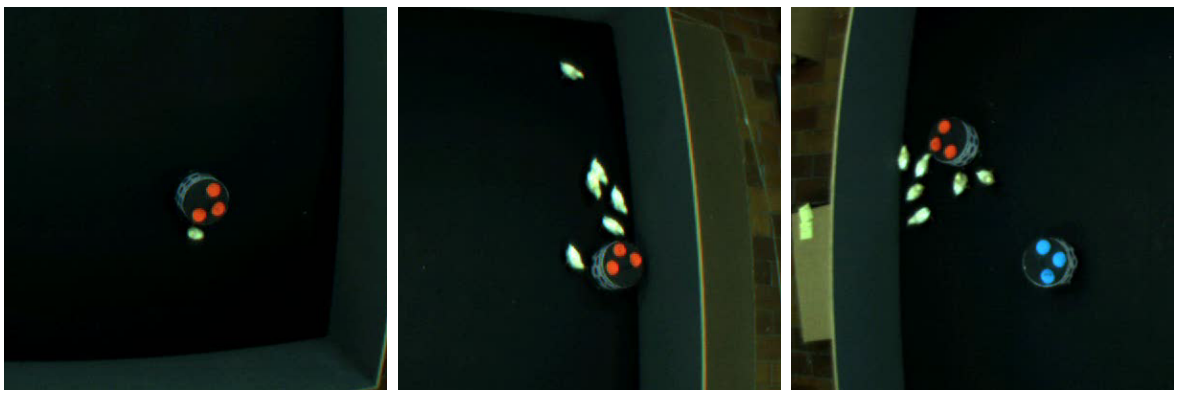

Figure 1: Three sample snapshots from videos taken with an overhead camera show chicks in individual and group experiments with one and several robots.

by the robot, and most experiments that we did were the "following" tests, where robots and chicks were released on the arena, robots were wandering on the experimental field and we observed the behavior of chicks. In experiments the robot receives information about its position and chicks positions in the real-time from the external vision system, and the acoustic information from the microphone array; this later can be mounted on the robot or used in the stand-alone mode. Output of the vision system is also displayed to the user in the intuitive way through monitoring tools. After the experiments precise trajectories of animals can be extracted by using the off-line tracker. Some elements of our experimental setup have been presented earlier in conferences, for instance, the design of the robot and the tracking system was discussed at [15], its audition module was presented in [16], finally, the safety system was reported at [17]. The goal of this paper is to give a comprehensive global view on the designed system, to provide a more detailed presentation of its elements' design, and to report experimental results on the validation of the social integration of the robot into the animal group.

The paper is organized as follows: Section 2 provides an overview of the experimental framework that we developed: an experimental arena with a monitoring system and the visual tracking system; in Section 3 we describe the mobile robot that we designed for experiments with chicks: its basic configuration, control system, and extension modules. Section 4 presents our efforts to 


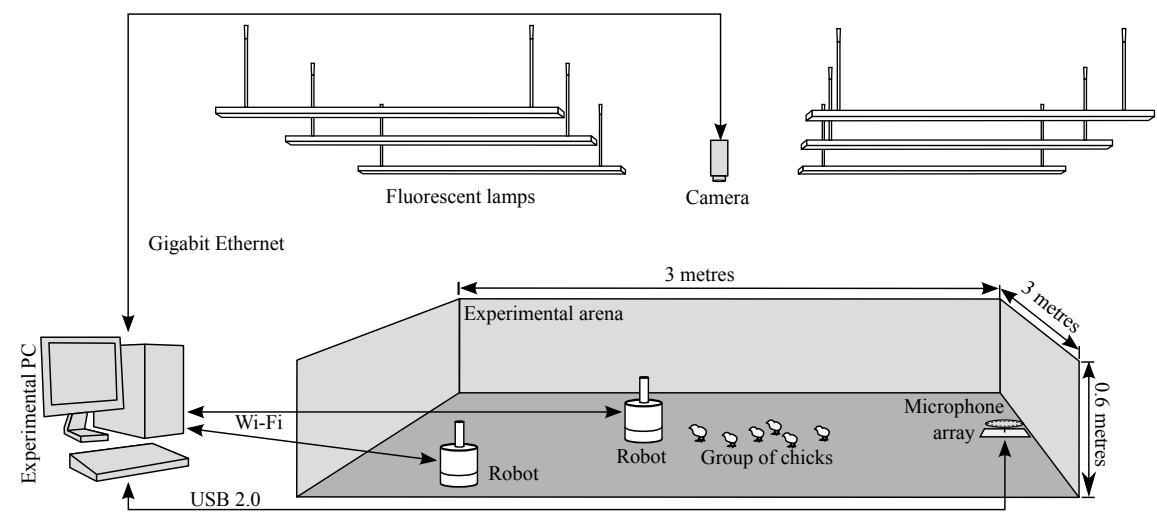

Figure 2: Overview of the experimental setup. Chicks (individually or in group) and one or two robots are placed on the experimental arena. Video data from the overhead camera and audio data from the microphone array are transferred to the experimental PC, where they are processed, and the results are sent to the robots.

make the robot safe. Section 5 presents results validating the social integration of the robot into the animal group and gives several examples of application of our system in behavior experiments. Finally, Section 6 concludes this paper.

\section{Experimental environment for interacting with domestic chickens}

In this section we present the experimental arena and the visual tracking system. An overview of the experimental setup is presented in Figure 2.

\subsection{Experimental arena}

The experimental arena is a flat square of $3 \mathrm{~m}$ by $3 \mathrm{~m}$, surrounded by a wooden wall of $60 \mathrm{~cm}$ in height (Figure 2). The floor is painted in black to simplify the tracking task. We run open-field tests, where animals and robots are released on the arena and their behavior is observed. The room temperature was kept constant at $22^{\circ} \mathrm{C}$.

To make the experiments possible it is crucial that the robot is accepted by the animals as a conspecific leader. The mechanism that helps us to achieve this goal is the filial imprinting mechanism. Imprinting is a special form of 
learning, during which a newly hatched chick may learn the characteristics of a parent. Imprinting occurs during a sensitive period that lasts for 5-25 hours after hatching [18]. Chicks have an innate tendency to develop a social attachment to the first moving object they see and to treat it as a parent [19]. To carry out the imprinting procedure, we place on the arena four wooden boxes $150 \times 15 \times 30$ $\mathrm{cm}$, where one wall is a transparent plexiglass. Each box has ten compartments of $15 \mathrm{~cm} \times 15 \mathrm{~cm}$ to house one chick. For the imprinting procedure, these boxes are placed on the experimental arena, two along one wall of the arena and two along an opposite wall, at distances to the walls of about $30 \mathrm{~cm}$, the transparent side facing the wall as shown in Figure 3. Thus, 40 chicks can be treated simultaneously. Imprinting sessions are done shortly after hatching: chicks are placed in the individual compartments, and robots move along the walls in front of boxes at a distance of about $100 \mathrm{~mm}$, forward and backward, at the speed of $60 \mathrm{~mm} / \mathrm{sec}$, one robot for every box. The robots emit calls and display a specific color pattern. As a call we used a calibrated sound with a frequency of $6 \mathrm{kHz}$, a beep duration of $150 \mathrm{~ms}$ and interval between two beeps of $350 \mathrm{~ms}$. These features have been chosen in correspondence to the range of chick audition $[20,21]$. We selected such an artificial sound to make this call unrelated to natural chick or hen calls, such as alarm calls or clucking; this was made for auditory imprinting not oriented towards a special message signal. The same call was played back in the hatching room during the incubation and hatching time.

The duration of each imprinting session is one hour, and it is repeated three times for every chick with one hour resting intervals. This type of imprinting setup corresponds to the state of the art $[22,23]$, and even goes beyond it in some characteristics. For example, in modern studies, computer screens are used to expose the imprinting object; we, on the other hand, provide a real agent that is moving and vocalizing. Besides this, the imprinting capacity of our device is higher, since each robot treats several chicks simultaneously, ten in our tests. The robot allows also to test the multi-modality of the imprinting learning by allowing the separate and programmable control of color, sound and 


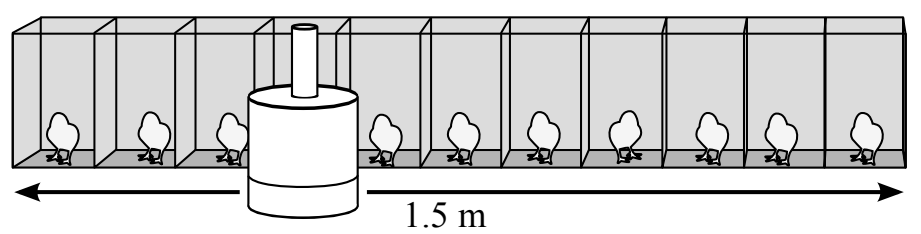

Figure 3: Design of the imprinting apparatus. Each box has ten individual compartments to house chicks. During the imprinting session robots move along the walls in front of the boxes, giving chicks a chance to observe it and to develop an attachment.

movement patterns.

\subsection{Visual tracking of animals and robots}

We use a Scout scA1000-30gc color camera by Basler Vision Technologies with a CS-mount T3Z2910CS varifocal lens by Computar. The camera is based on a Sony CCD sensor and has a resolution of $1032 \times 778$ pixels and a maximum frame rate of 30 frames per second. Twelve lamps were uniformly fixed on the ceiling to provide lighting conditions as homogeneous as possible. A common daylight lamp is a source of a strong infrared (IR) emission that affects the IR sensors of the robot. To resolve this issue, we used lamps with reduced infrared emission FQ49W/965 by OSRAM.

The camera uses a Gigabit Ethernet connection to transfer video to the PC. The experimental PC has an eight-core Intel Core $^{\mathrm{TM}} 2$ Quad processor and $2 \mathrm{~GB}$ of RAM. It runs the monitoring and recording software and the Graphics User Interface module of the robot control system. It also serves as a temporary storage of the experimental data recorded during the day, before the data is transferred to external hard drives.

We used two different approaches to track chicks - one solution for a realtime tracking that provides only positions of chicks without keeping individual trajectories and another solution for an off-line data analysis that extracts trajectories for every animal. Two different techniques were used for several reasons, first of all, during the experiments what we need to know are positions of robots and animals to make the robot respond to the group displacement; 
in the same time reliable individual trajectories are less important here. In contrast, for understanding individual behaviors the trajectories are essential as they form the basis for further analysis. Another reason is the complexity of the fully automated tracking in cases when simultaneously several chicks are hidden occasionally behind the robot during an experiment that makes the reliable automatic trajectory association a difficult task, and thus an option to correct results manually is needed.

For real-time tracking we used the SwisTrack software. SwisTrack is an open source component based cross-platform tracking software initially developed to track cockroaches and robots [24]. For image processing, SwisTrack relies on the OpenCV computer vision library [25]. The routine to track robots and chicks displacements is presented in Figure 4a. The robot position and orientation are defined by three color markers on top of it (see Section 3). Markers can be detected by subtracting the color of the markers from the input image and binarizing the result with a predefined threshold. The same approach is used to track the positions of individual chicks at each video frame. We also estimate the group position as a whole, that is, the group center of mass and size. Several appropriate components were implemented to the SwisTrack to do this task. Estimated robot position and orientation, chick positions and group parameters are transferred to the robot control system through the wireless radio connection. SwisTrack is also used for recording the experiments.

In the off-line tracker for the image segmentation we use the Gaussian mixture model (GMM) based clustering approach that is one of the popular techniques for image segmentation; the variational Bayesian approach used for training is more robust than the classical maximum likelihood (ML) method that suffers from the over-fitting problem in cases when some chicks are accidentally hidden behind the robot. The variational Bayesian approach handles this problem by adapting the number of components and pruning the components that are not used $[26,27]$. It is also free from the singularities problem of the ML-based training approach. Figure 5 demonstrates the result of the sample experimental image clustering by using the variational Bayesian GMM approach. 


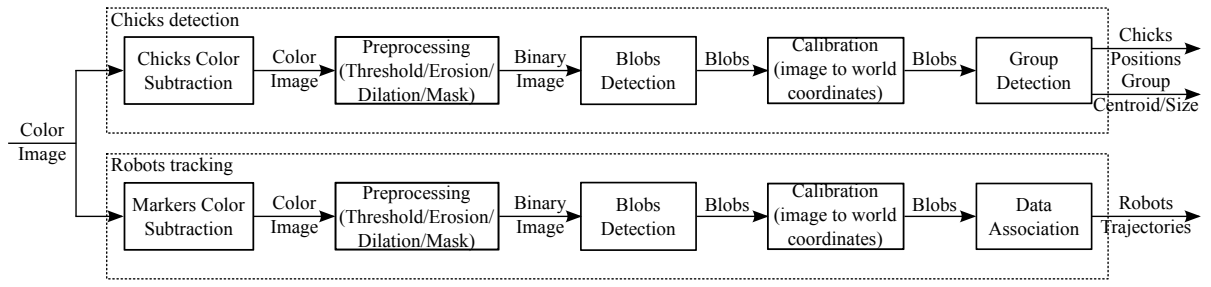

(a) Data flow in the real time vision system. The robot position and orientation are defined by three color markers on top of it. Chick detection is also color based. Estimated robot position and orientation, chick positions and group parameters are subsequently sent to the robot control systems.

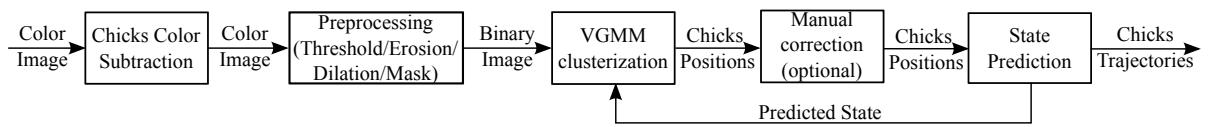

(b) Data flow in the off-line tracker. The variational mixture of Gaussians approach is used for the image segmentation, and particle filters are used to initialize mixture parameters. If necessary, tracking results can be manually corrected.

Figure 4: Tracking overview. Two approaches to track chicks are used: a solution for a real-time tracking and another one for an off-line trajectories extraction.

The initial values for the parameters are chosen, as suggested in [26].

A well-known limitation of the GMM based clustering lies in the mixture parameters initialization procedure: to get good segmentation results, we must provide reliable initial conditions. Moreover, while detecting positions of chicks, the clustering provides no information about their behavior in time (i.e. no data association). Hence, it is desirable to combine the clustering with some method able to track chicks by probabilistically integrating all measurements available up to the current time. It was shown that particle filters provide an effective way to track acoustic and visual targets [28, 29]. Using a sequential Monte Carlo method, particle filters recursively estimate the probability density of the unknown source state conditioned on all the received data up to and including the current frame.

At time $t$ we model every chick $c=0,1, \ldots, C-1$ by using $P$ particles at positions $\mathbf{x}_{c, p}^{(t)}$, each with its weight $w_{c, p}^{(t)}$. The state vector for particles has four 

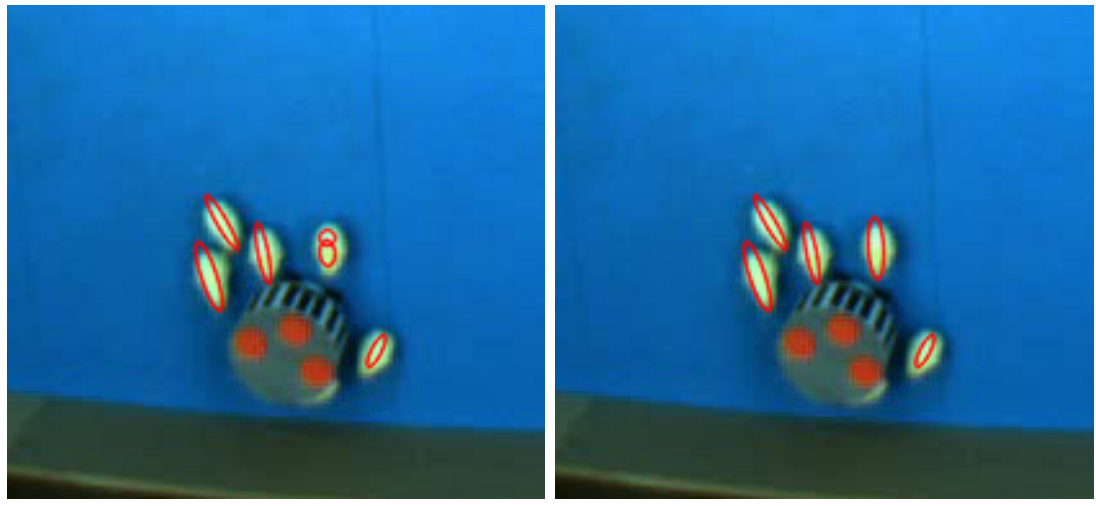

Figure 5: Detection of the chick positions. On the left: the GMM clustering suffers from over-fitting when some chicks are not visible. On the right: a variational Bayesian GMM automatically adapts the number of components in the mixture.

dimensions, two for position and two for speed:

$$
\mathbf{s}_{c, p}^{(t)}=\left[\begin{array}{c}
\mathbf{x}_{c, p}^{(t)} \\
\dot{\mathbf{x}}_{c, p}^{(t)}
\end{array}\right] .
$$

We use the sampling importance resampling algorithm with a predictor in the form, used in [28], as it was shown to work well in practice. The model is defined as

$$
\dot{\mathbf{x}}^{(t)}=a \dot{\mathbf{x}}^{(t-1)}+b F_{\mathbf{x}}, \quad \mathbf{x}^{(t)}=\mathbf{x}^{(t-1)}+\Delta T \dot{\mathbf{x}}^{(t)},
$$

where $a=e^{-\alpha \Delta T}, b=\beta \sqrt{1-a^{2}}, F_{\mathbf{x}}$ is a normally distributed random variable and $\Delta T$ is the time interval between two frames. The positions of the chicks, predicted by the filters, are used as initial means values of the Gaussians for the clustering procedure. The tracking routine is shown in Figure 4b. Our tracking approach is similar to ones used to track flies in off-line [30] and fish in on-line [10] tracking software.

For about $0.7 \%$ of the frames, the tracker fails to associate chicks to the corresponding trajectories; usually this happens when several chicks form a dense group or are simultaneously hidden behind a robot. In such cases, tracking results had to be corrected in a manual mode that is specially provided in the tracker for this purpose. 


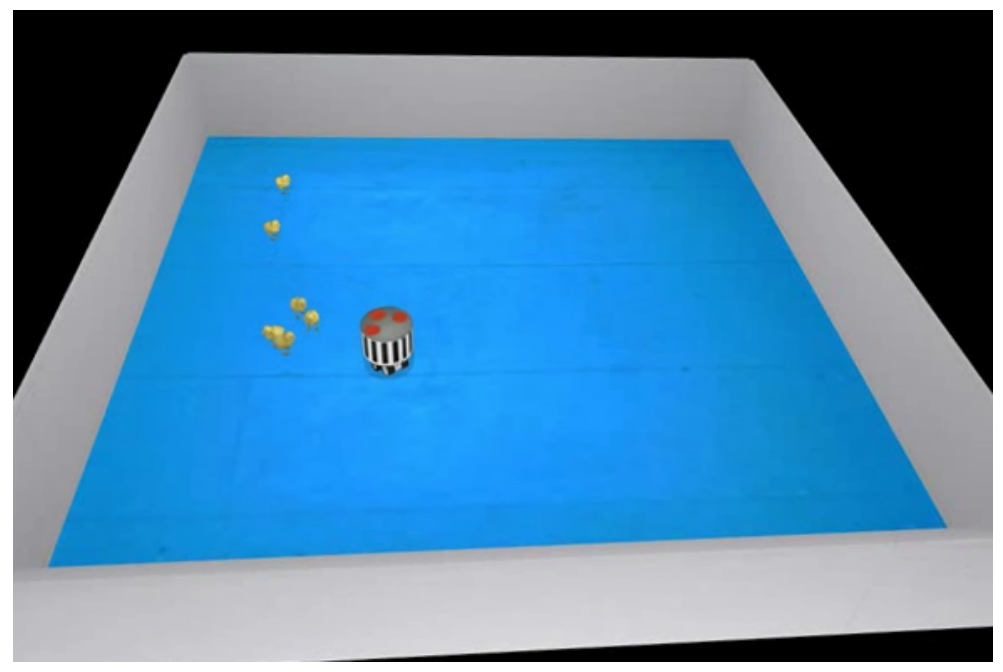

Figure 6: The remote monitoring interface gives a virtual representation of experiments in real time.

The coordinates of the robots and the animals extracted from the video were mapped to the real-world coordinates (in $\mathrm{mm}$ ) by using the calibration routine based on the Tsai's calibration technique [31]. For our setup, the average absolute calibration error is $3.1 \mathrm{~mm}$ and the maximal calibration error is 6.1 $\mathrm{mm}$.

\subsection{Remote viewer to monitor experiments in real time}

To avoid bias in the behavior of the animals, nobody is allowed to be in the experimental room while the experiments are running. To remotely observe a situation on the arena, we developed a lightweight and simple multi-platform remote viewer that connects to the SwisTrack through TCP/IP and provides a $3 \mathrm{D}$ representation of the arena (Figure 6 ). The data stream is coded by using the NMEA 0183 protocol; since only coordinates are transferred, the load on the network is considerably lower than if we would transfer a video stream. An useful feature of the remote viewer is an embedded robot failure detection module presented in Section 4.2. 


\section{The PoulBot robot}

In this section, we discuss in detail the hardware design of the PoulBot robot, its control system, and the audition system designed for the robot.

\subsection{Hardware design}

The design of the body and the behaviors of a robot dedicated to experiments with animals have to take into account relevant sensory modalities and behaviors of the animal under study [32]. As it was mentioned, to make the robot accepted by the animals we used the filial imprinting mechanism. The primary tendency to follow is inherited by chicks, but they learn what to follow. Still, there are inherited constraints on what they may learn: chicks are more likely to imprint if the object is about the size of a hen than if it is much smaller [18]; they prefer the patterned object to the plain [33], and the moving objects are more attractive than the stationary [34]. Chicks are likely to be imprinted if the color of the object is strongly distinguishable from the background, with red and blue to be the most attractive colors [35]. Auditory stimuli are also of high importance for imprinting; the chicks are more likely to imprint if the object emits calling sounds than if it is silent [36].

Based on this results, in the beginning of this project we draw up the following specification for the robot: dimensions of the robot have to correspond to the dimensions of an adult chicken; it has to be able to travel in an environment conducive to chicken experimentation, indoor or outdoor, considering droppings; the battery runtime has to be up to several hours, depending on the type of the experiment. The robot has to carry a color pattern, to be capable of emitting a sound from a library of available sounds and to be capable of detecting and reacting on sounds emitted by animals. Finally, the robot has to provide wireless communications with a central fixed processing unit.

The robot that we built and named PoulBot is presented in Figure 8. This track-type mobile robot is a configuration of the marXbot robot [37] - a modular research robot developed at EPFL. Thanks to its modular structure, the 


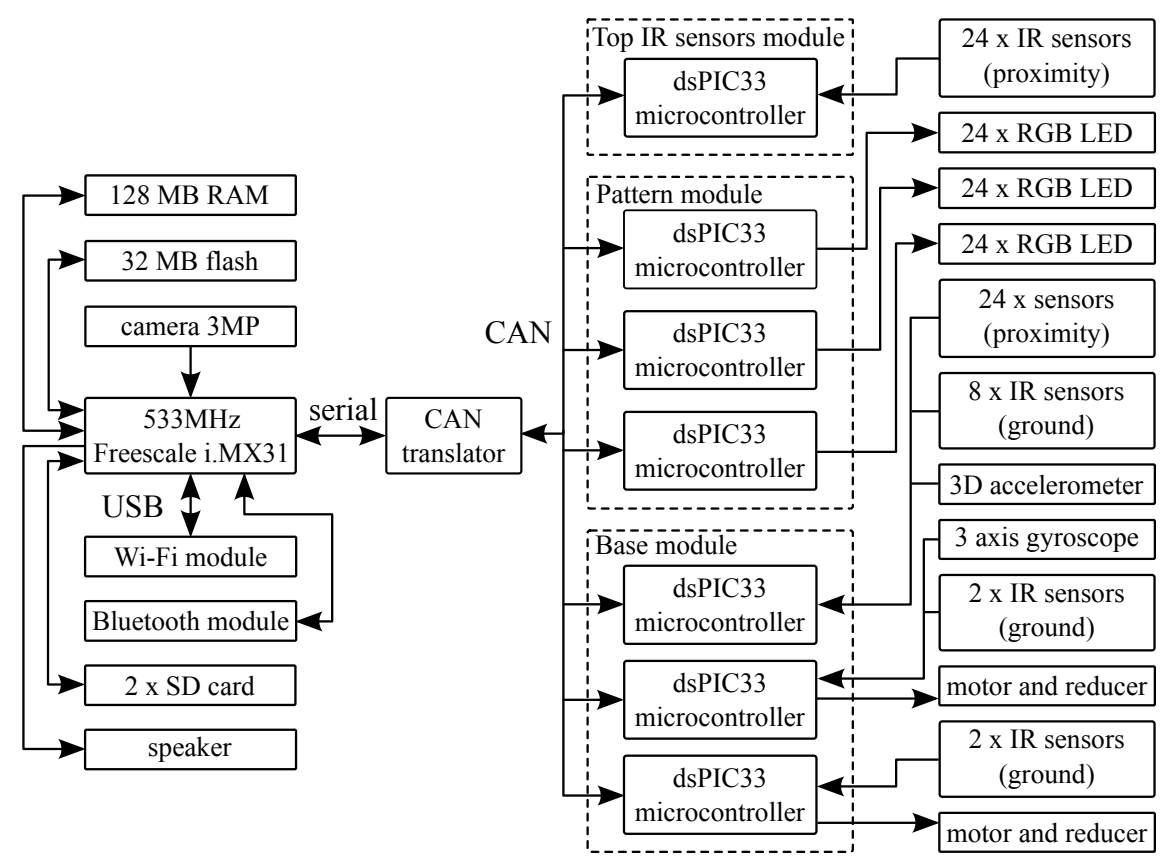

Figure 7: Overview of the hardware architecture of the PoulBot robot.

marXbot can be adapted to various research projects: we can remove modules that are not needed, and we can also develop new modules, depending on the specifications that the robot has to fulfill. For the PoulBot robot we used two modules of marXbot: a base module providing energy, means for locomotion, motion and rotation sensors, short-range proximity sensors and a speaker, and a main computer board featuring an ARM processor, Wi-Fi and Bluetooth modules, a speaker, and an omnidirectional camera. The following modules were developed in our project: a pattern module allowing a robot to carry a specific pattern, a plexiglass bumper providing passive protection to chicks, an extra ring of proximity sensors placed above the color pattern module, and a microphone array. On the top of the robot we fixed three color markers used to track the robot position and orientation as it will be shown below.

Every module of the robot consists of one or several Microchip dsPIC33 microcontrollers that drive the sensors and/or actuators of the module (Figure 


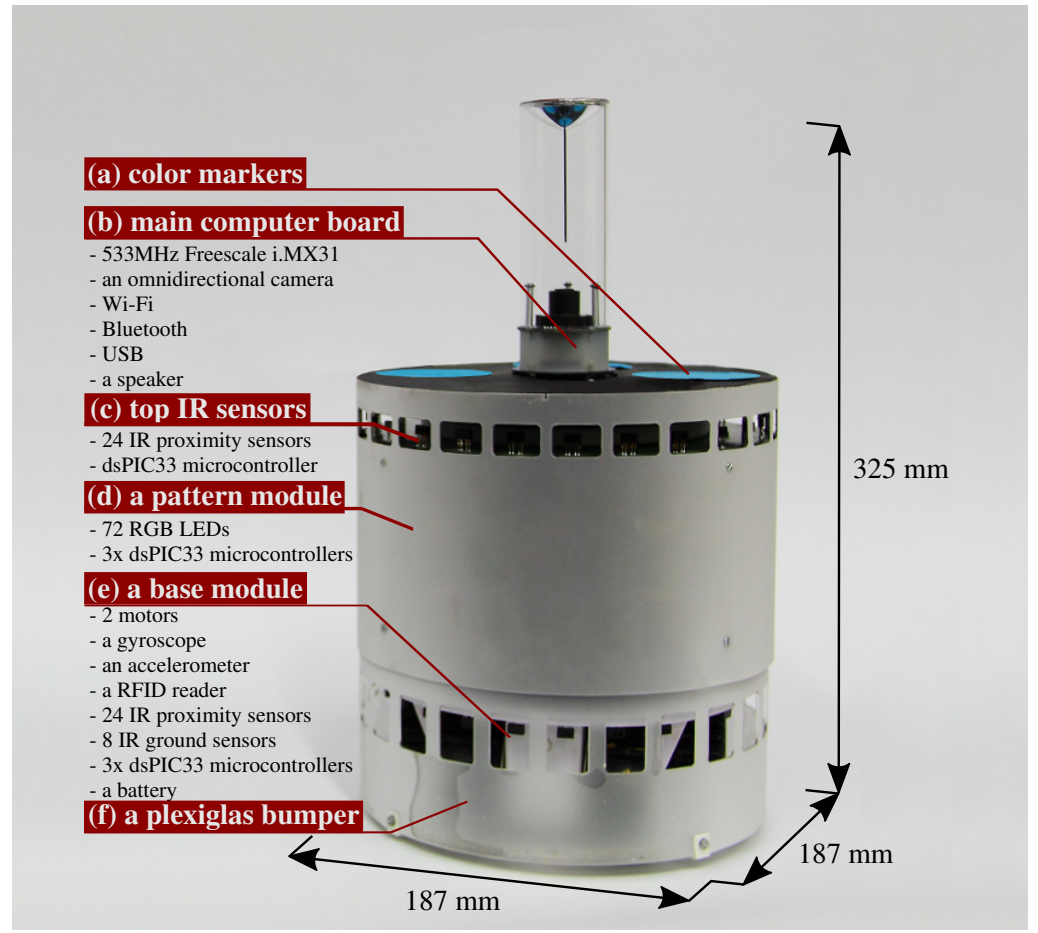

Figure 8: The basic configuration of the PoulBot robot consists of six modules providing the robot with locomotion, sensing and communication capabilities.

7). The microcontrollers have a processing power of 40 MIPS and are connected with each other and with the main computer of the robot through the controllerarea network (CAN) bus [38] that supports both multiple masters and masterslave mode and provides velocity up to $1 \mathrm{Mbit} / \mathrm{s}$. Such a distributed processing allowed us to achieve a real parallelism of control, based on an event-based communication among modules [39].

The standard configuration of the PoulBot robot is presented in Figure 8 and consists of the following modules: the base module, the bumper, the pattern module, the main computer board, the wall detection module; the microphone array can be additionally installed on the robot.

The base module has two treels - a combination of tracks and wheels that guarantee stable contact with the ground and remarkable maneuverability. The 
module is also equipped with a gyroscope, an accelerometer, an RFID reader, 24 short range infrared proximity sensors around the robot used to detect and avoid obstacles, and eight infrared ground sensors facing down. The power is provided by a replaceable $38 \mathrm{Wh}$ lithium polymer battery. To protect chicks from the tracks of robots we use the plexiglass bumper. The bumper is not transparent so as to prevent the chicks from been imprinted on the wheels especially since the bumper is a part of the robot that is at eye level of a young chick. It has windows for IR sensors.

It was said above that a pattern placed on the imprinting object improves the imprinting results. A plastic tube is mounted above the base module, it envelopes the top IR sensors module. A paper color pattern can be fixed on it and was used in first experiments, later, to have a possibility to change color pattern dynamically we developed a RGB LEDs pattern module inside the tube. In this latter case the plastic tube serves as a light diffuser. The module consists of three rings of RGB LEDs mounted one above another and a nontransparent replaceable pattern tube. Every LEDs ring consists of 24 diodes that are driven by a dsPIC33 microcontroller and isolated from one another by nontransparent horizontal and vertical partitions to clearly separate the light sources. The intensity and color of every LED can be set separately, thus allowing the production of a variety of colors and spatial combinations (Figure 9). Thanks to the replaceable pattern tube, different shapes of pattern elements can be tested. This technique is similar to the approach used in studies on cognition abilities in domestic chicken, where LCD screens are used to show different shape and colors $[23,40]$.

We needed the robot to be capable of moving safely on the arena, minding walls and chicks. The IR sensor ring at the base was not enough since it informed us about all types of obstacles (walls and chicks), and such was not desirable since we wanted the robot to react differently on their presence. To address this limitation, an additional short range proximity infrared sensors ring with 24 sensors was placed on the top of the robot. Being high enough to guarantee that it does not perceive the chicks, this ring is used to detect and avoid walls. 

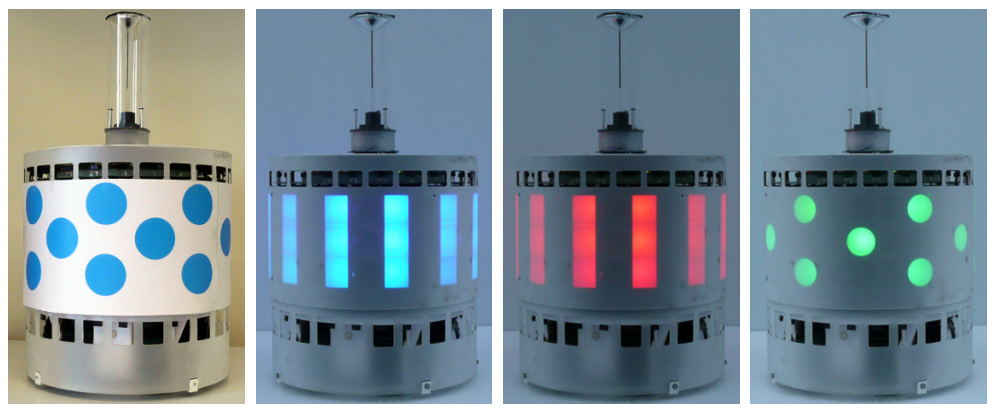

Figure 9: A color pattern is placed on the robot to improve the quality of imprinting. A paper color pattern (on the leftmost image) can be used in the simplest case, alternatively we can use a RGB LEDs pattern module to produce various color patterns that can be dynamically changed.

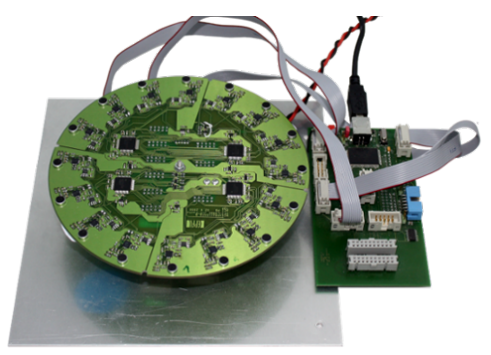

Figure 10: The microphone array and the acquisition board. Here the microphone array is mounted on the support to be used separately from the robot.

The distributed microcontrollers of the robot efficiently cope with managing the motors and most of the sensors, as well as with running low level behaviors. But for higher level activities (e.g., mapping, localization, path finding, or navigation) and to manage sensors demanding a significant computational power (such as vision or audition), a more powerful processing unit is needed. The PoulBot main board central computer is a 533MHz Freescale i.MX31 with 128 MB of RAM. It runs Linux 2.6.33; the communication with microcontrollers is carried out through the translator that links CAN network with a serial port of the i.MX31. A three megapixels camera facing up is connected to the central computer and provides an omnidirectional vision. The main board also provides Wi-Fi and Bluetooth wireless connectivity. 
To endow the robot with a capability to localize several sound sources we developed an audition module for the PoulBot robot. It consists of a microphone array and the acquisition board that can be mounted on the robot or used separately. The circular array consists of 16 microphones and has a diameter of $15 \mathrm{~cm}$ that corresponds to the diameter of the robot (Figure 10). The uniform circular array geometry was chosen, since comparing with the linear array geometry it is free of such limitation of a latter one as left-right ambiguity, non-unique direction vectors resulting in grating lobes, and non-uniform resolution over the entire horizon [41]. When selecting number of sensors we considered two variants: 8 and 16 microphones, since with more microphones the computational burden would be too heavy for the main processor of PoulBot. The analysis of squared aperture smoothing function $|W(\vec{k})|^{2}$ and of maximum sidelobe level function $[42,43]$ showed that the array of 16 microphones has significantly better anti-aliasing properties than the array of 8 microphones (see Figure 11 for details). In the acquisition board we use a Cypress FX2 host controller for data acquisition and transfer; the board is capable of sampling 16 microphones synchronously, with 16-bit resolution and sample frequencies up to $50 \mathrm{kHz}$. The data acquisition is done in real time and the acquired data is transferred via USB 2.0 High-Speed. The board can be connected directly to the USB port of the i.MX31 main board of the robot or to the external PC [16].

The robot lifespan without recharging is about one hour if the RGB LEDs pattern module is used and up to three hours without it. That in principle could limit the maximum experiment duration, but thanks to the marXbot's multibattery recharging station developed for the collective robotics experiments [37] and the robot hot swap capability, the battery can be automatically replaced during the experiment.

Four PoulBot robots were built thus we could run multi-robot experiments and to have several spare robots. 

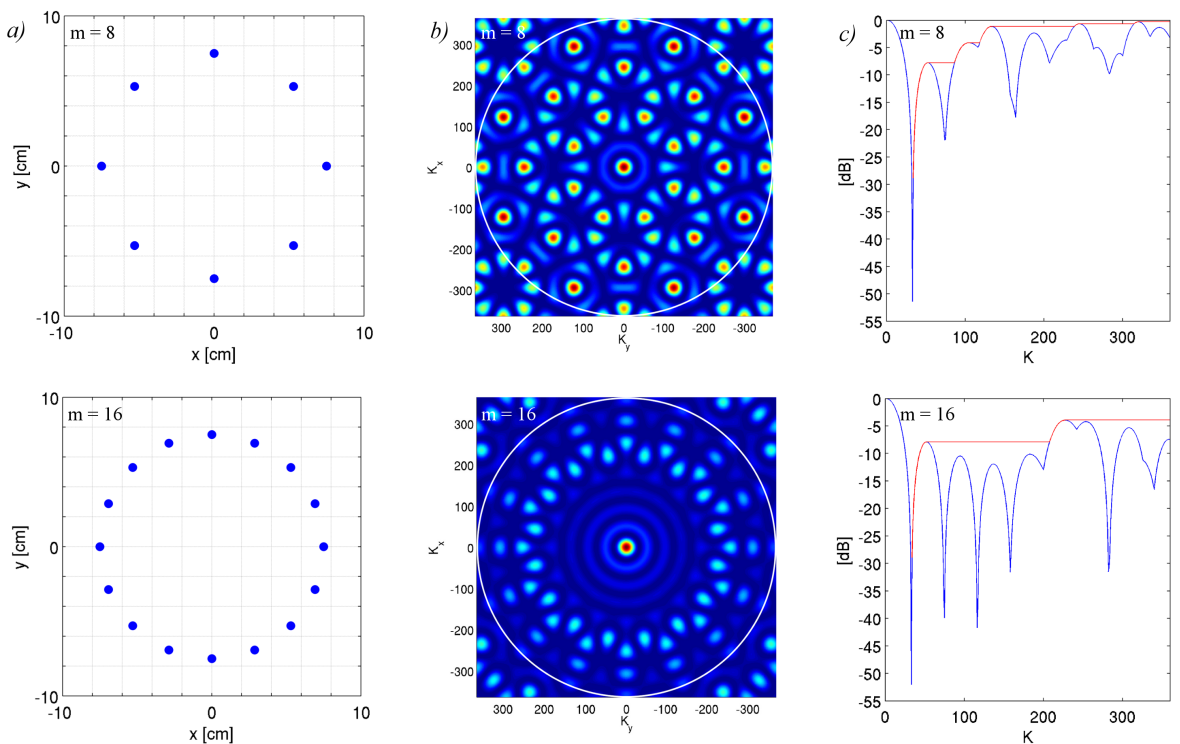

Figure 11: Design of the PoulBot audition system, comparing cases of 8 and 16 microphones. From left to right: (a) sensors locations on circular arrays of 8 and 16 microphones; (b) values of $|W(\vec{k})|^{2}$ for two cases, here $W(\vec{k})$ is the aperture smoothing function and the vector $\vec{k}$ is the wavenumber vector those direction corresponds to the direction of a plane wave propagation and $|\vec{k}|=2 \pi / \lambda$, where $\lambda$ is a wavelength [42]. Here $f_{\max }$ is taken to be equal to $10 \mathrm{kHz}$ and the white circle corresponds to wavenumber vectors up to $2 k_{\max }=2 \frac{2 \pi f_{\max }}{c}$, where $c$ is the speed of sound [43]. The mainlobe is given by $W(0)$, we can also observe a number of sidelobes (smaller amplitude peaks not located at zero wavenumber). Sidelobes correspond to directions from which we will pick up an interference while listening from a given direction. It can be seen that the array of 16 microphones has significantly better anti-aliasing properties than the array of 8 microphones array, as the latter has ghost peaks whose values are almost equal to the mainlobe. (c) The radial profile (blue) and maximum sidelobe level (red) functions characterize anti-aliasing properties for two microphone arrays [43]. It can be seen that for 16 microphones, a ratio between the mainlobe and sidelobes is around 2.5 . 

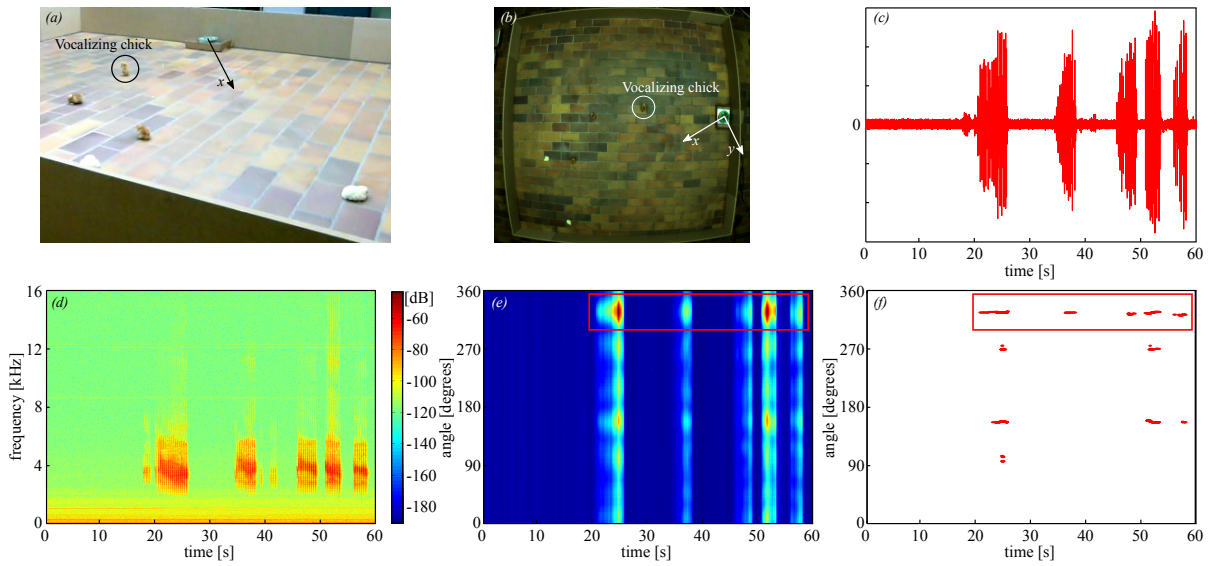

Figure 12: The localization of a calling chick by a microphone array. (a) The view of the arena from the side and (b) an overhead camera. (c) The call waveform. (d) The call spectrum. (e) The result of applying the sound localization system. The rectangular marks a chick producing calls. (f) The detected sound sources directions, corresponding to maximal peaks of the beamformer output energy. The sound sources directions due to reflections can be filtered out, as it is described in [16].

\subsection{Sound source localization with a microphone array}

As was mentioned, vocal communication plays an important role for birds. The principal sound signals of the domestic fowl chicks, as well as for youngsters of many other species of birds, are the calls of distress and of pleasure; these calls reflect a state of security or insecurity felt by the chick [20]. Thus to react to such calls the robot has to be able to detect them and to localize chicks that call.

In last two decades acoustic arrays have been used in biology to localize wild-life animals and monitor their interactions; for an overview see [44]. In such applications usually the large array are used distributed in the wild that allows to provide a more detailed and precise data. In robotics, the field of robot audition is still in its infancy, it is far behind artificial vision. However, currently, this field is a subject of much research [28]. The most advanced of the modern robotic audition systems, HARK by Kyoto university [45], however, to work in real time, this system demands a modern multicore processor with a 
sufficient amount of RAM which is not available for the moment on small mobile robots. There were attempts to adapt advanced acoustic methods to run on DSP [46], but the resulting performance was significantly below the original one. For this reason, less computationally expensive methods are usually used in audition systems for mobile robots. For example, a delay and sum beamforming is a common method for sound sources localization $[47,48]$. In the sound localization systems, based on the beamforming approach, microphone arrays are steered to multiple directions and the maxima of output signal energy are then searched. An advantage of this approach over other sound localization methods, such as for example those based on time-difference of arrival information or techniques adopting spectral analysis concepts, is that beamforming can be used for wideband signals and multi-speaker cases [49].

We use the interpolation beamforming - a modification of the delay and sum beamforming method [50]. Let $x_{m}(t)$ be an input signal for the $m$-th microphone, $m=1,2, \ldots, M$, and let $\theta$ be an arbitrary direction in the plane of the microphone array, $\theta \in[0,2 \pi)$. The output of the classical delay-and-sum beamforming steered to the direction $\theta$ has the following form:

$$
y^{\theta}(n)=\sum_{m=1}^{M} x_{m}\left(n-\hat{\tau}_{m}^{\theta}\right),
$$

where $\hat{\tau}_{m}^{\theta}$ is an approximation in sample units of $\tau_{m}^{\theta}-$ a time of arrival difference between the $m$-th microphone and the array centroid. The output energy of the beamformer steered to direction $\theta$ over a frame of the length $N$ is given by:

$$
E(\theta)=\sum_{i=1}^{N} y^{\theta}(i)^{2} .
$$

If a sound source is located in direction $\theta^{\prime}$, then the beamformer output energy $E(\theta)$ will have the local maximum at the point $\theta^{\prime}$.

The main drawback of the delay-and-sum beamforming lies in the sampling procedure: to approximate time delays $\tau_{m}^{\theta}$ required for beam steering with a reasonable precision, signals have to be sampled at a rate much greater than the Nyquist frequency. The interpolation beamforming method overcomes this 
constraint by temporally interpolating the sensor data before the beamforming operation. It allows the sampling of input data at Nyquist rate. The interpolation is performed by zero padding the sensor data and passing the resulting sequence through a finite impulse response (FIR) low-pass filter.

To guarantee that all microphones of the array have the same gain we use the microphone gain self-calibration procedure proposed in [51]. The procedure is based on a projection of microphone coordinates on the direction of sound arrival line, followed by an approximation of received energy levels.

\subsection{Robot control}

To build the control system of the PoulBot we used a behavior based approach [52]. Behavior based systems are known to work well in dynamic environments where fast reaction and high adaptability are important. These characteristics make the behavior based approach a natural choice when designing a control system for a robot that interacts with animals. The PoulBot is equipped with a set of primitive behaviors tightly bonded with the sensors and actuators of the robot. Each primitive behavior serves to achieve a particular goal or to perform a specific activity. Examples of primitive behaviors are "follow-wall", "emit-sound", "go-to-target", etc. These behaviors are executed on the microcontrollers of modules of PoulBot. Some primitive behaviors can be executed in parallel, for instance, "follow-wall" and "emit-sound" behaviors, while others might produce conflicting commands, such as "avoid-obstacle" and "go-to-target" behaviors. In such case we use the priorities base arbitration [53] to select the primitive behavior to be executed (Figure 13). Some primitive behaviors in addition receive information on robot's position and on positions of chicks from the external vision system described in Section 2.2 through the wireless connection (Wi-Fi or Bluetooth), for instance in the "go-to-target"

primitive behavior robot's position is used to compute a heading and a distance to the goal.

Poulbot's primitive behaviors are combined to generate complex behaviors necessary for specific experiments. Complex behaviors can run on the robot 
main board, or on the experimental PC (for debug and demonstration reasons). On the Figure 13 we show an example of the complex behavior called "wandering". It was used in the experiments where we studied the robot following dynamics of the animal group (see Seciton 5.2 for details). When executing the "wandering" behavior, the robot with an activated pattern and emitting a sound travels on the experimental arena. It goes straight by executing the "goforward" behavior until it meets a wall, then the "avoid-wall" behavior with a higher priority is activated and the robot turns against the wall and then again goes straight. Besides, the chick safety behaviors are activated that are described in details in the next Section. A number of complex behaviors were implemented, among them the "imprinting" behavior used in the imprinting procedure, the "remote-controlled" behavior that allows the user to control the robot very much as a toy car, the "bring-chicks-to-targer-behavior" that serves to carry out the group following with feedback experiment that we will describe in Section 5.2.

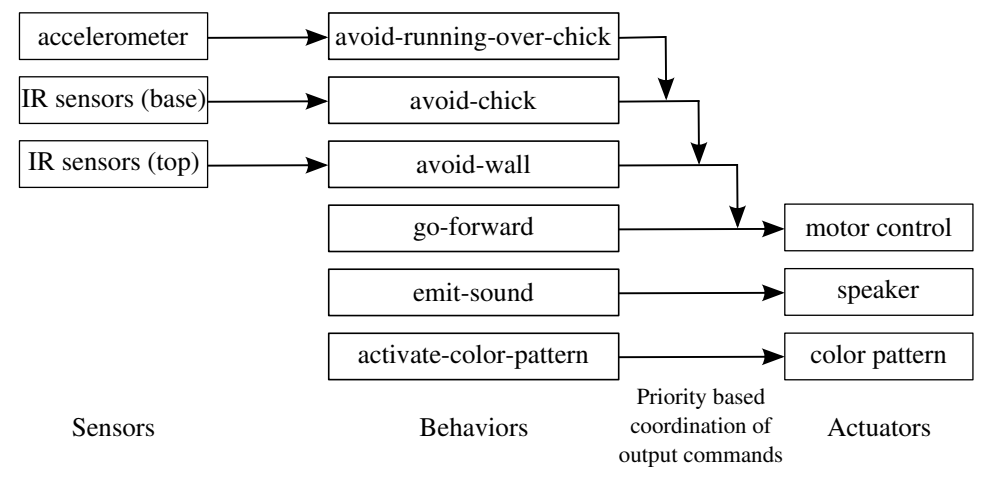

Figure 13: Six primitive behaviors are combined to generate the complex "wandering" behavior. The primitive behaviors are sorted in the order of priority, the behavior on top has the highest priority. Among the competitive behaviors the priority-based arbitration is used that allows to give the behavior with the highest priority the control of the actuators.

Complex behaviors are activated by the user in the dedicated graphical user interface (GUI) that runs on the experimental PC; it is presented in Figure 14. This GUI also allows the user to specify parameters affecting behaviors (e.g., 
speed, sensors thresholds, etc.) as shown in Figure 15; for instance for the "wandering" behavior the user can set the following relevant parameters: robot speed, the color pattern used, sound modulation, accelerometer threshold. The GUI also provides the user with the information on the status of the robot, such as battery charge level and current executed behavior.

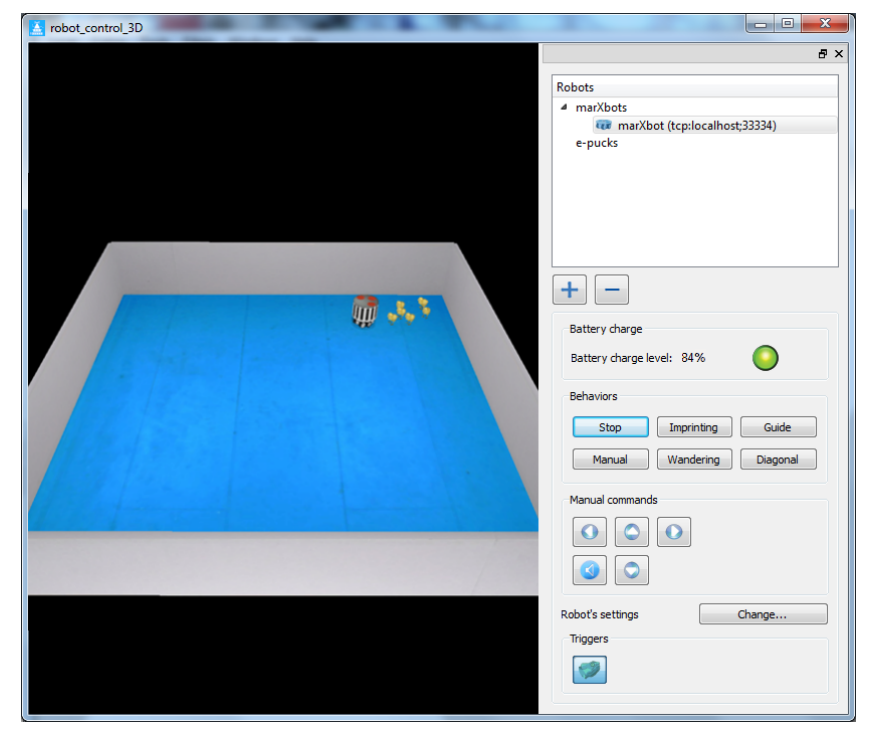

Figure 14: The graphical user interface of the PoulBot control system. On the left side is the virtual view of the experimental arena that receives data on robot's and chicks' positions from the external vision system, while on the right side is the list of connected robots and the control elements of the selected robot that allow the user to activate a specific behavior and to specify its parameters.

\section{Safety measures for animal experiments}

In experiments where animals and robots share the same physical environment, there is always a risk of a robot accident that can lead to animal injuries. Besides fundamental ethical reasons, in the case of an accidental injury or death of an animal before the end of experiment, the data collected on this animal most of the time cannot be used for further analysis as it is not complete; in the case of the experiments on the collective and especially social behavior, when 


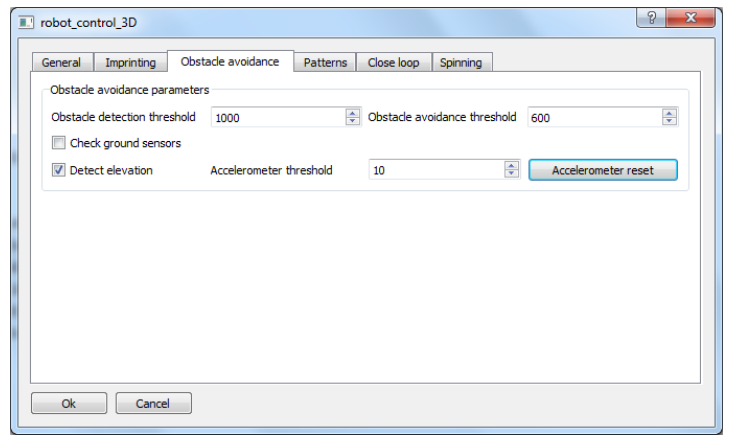

Figure 15: The graphical user interface provides an access to parameters of robots behaviors such as speed, thresholds for different sensors, etc.; the parameters are logically grouped in several tabs.

animals are observed in groups, it is even worse, since in this case the whole group is impaired and has to be removed from further tests. Thus in projects where the robotic devices are used in biological studies, safety becomes a key issue. However the topic of robotic safety in experimental biology has not been given sufficient attention yet, to best of our knowledge there are no studies that addresses this issue. In our work to design a safe robot we adapted the safety recommendations originating from the field of physical human-robot interaction [54]. The safety elements were introduced in the mechanical design of the robot, its control system and into the experimental environment.

\subsection{PoulBot on-board safety systems}

To make PoulBot safer for chicks, we used a combination of several passive and active safety measures. First of all, in the mechanical design, the plexiglass bumper was used as a passive mechanical protection (Figure 8). The tracks of the robot are very efficient on the rough terrain, but they constitute a danger to the chicks since chicks have relatively long toes which can get stuck between the track lugs. This could lead to injuries, or even to make a robot run over a chick, and such can be life-threatening. The plexiglass bumper reduced the potential for wounds. 
Second, safety behaviors were implemented that rely upon the proximity sensors and the accelerometer at the robot base to detect and avoid collisions. For a collision avoidance the "avoid-chick" behavior was added that serves to reduce the risk of collision with a chick and to minimize the eventual effects of such a collision. This behavior uses the data from the IR sensors of the base and top modules; these sensors have a short range: the values that they provide are reliable up to $3 \mathrm{~cm}$. As an obstacle avoidance technique we use the vector field histogram (VFH) method [55]; in this method, once the IR sensors of the robot detect an obstacle, the sensors data is used to create a local occupation polar histogram. A threshold value is used to determine the obstacles' free directions. Then, a direction closest to the original moving direction is selected and the corresponding steering angle is set. This behavior runs on the microcontroller of the base module responsible for driving the IR sensors.

The second safety behavior is called "avoid-running-over-chick" and complements the "avoid-chick" behavior in those cases when IR sensors do not detect a chick, for instance, if a chick has fallen asleep during the experiment and hence lies below the level of the sensors. In such cases, a collision risk grows and there is the possibility that the robot will run over the chick. The "avoidrunning-over-chick" behavior was designed to reduce the potential injuries of such a collision. It uses the accelerometer readings to detect when a robot is not on a flat ground anymore. In this case, it makes the robot to roll back and to turn away from the initial direction of movement. Safety behaviors have a higher priority with resepct to standard experimental behaviors.

Still sometimes the robot can be unaware that his behavior is not normal anymore, due to the fact that it has access only to the local information. In this an external failure detection system can be used that observes the situation on the arena and interrupts the experiment if a non-planned situation happens. Such a system is presented in the next section. 


\subsection{External failure detection systems}

In Section 2.3 we presented a remote viewer that connects to the SwisTrack through TCP/IP and provides a 3D representation of the arena. An additional plug-in to this viewer was developed that automatically analyses the experimental situation to detect abnormal situations. This can be done thanks to the typical predictability and repeatability of the robotic behavior in the animal experiments, guaranteeing that experiments can be repeated many times in the same conditions, potentially allow us to replace a human observer by a trained automatic classifier. The idea is to train the classifier to automatically recognize behaviors executed by the robot, in this case the unexpected behavior (due to an accident or malfunctioning of the robot, for instance) will be considered as an outlier and the system has to warn the user. For this purpose we used the novelty detection technique based on the extreme value theory (EVT), introduced in [56]. This technique uses a Gaussian mixture model to represent the data distribution, then it examines the distribution tails and estimates the probability that a given instance is a extreme value in the distribution model. To train the model to recognize behaviors the following parameters can be used: duration of the behavior, mean linear speed of the robot, mean absolute rotation speed of the robot, mean distance from the robot to the closest wall, etc. More detail on the system and experimental results can be found in [17].

\section{Experiments}

In this section we demonstrate that the PoulBot robot can be successfully integrated into the animal group and give several examples of application of our system in behavior experiments.

\subsection{Validation of social integration}

As it was mentioned earlier, there are many factors that can affect the quality of imprinting. We want to show that the imprinting mechanism allows the social integration of the PoulBot robot into the animal group. After the imprinting 
procedure every chick was tested individually in the "following" test. Every test was recorded, then an expert looked through videos and labeled the chicks according to their behavior. A chick was considered to be imprinted on the robot if it stayed close to the robot and follow it around the arena during the experiment. Out of 212 chicks 126 were labeled as imprinted resulting in the imprinting rate of $59.43 \%$ that is comparable and even exceeds the results reported in the classical studies on imprinting $[18,57]$.

To show that the phenomenon of the chicks following the robot is related to the imprinting procedure we compared the reaction on the robot of chicks in the control groups that did not undergo the imprinting procedure with the reaction of chicks from experimental groups that had the imprinting sessions. We analyzed 60 following experiments done with 30 experimental groups and 20 experiments with 6 control groups. Figure 16 gives two examples of arena occupancy grids corresponding to a typical control group and to a typical experimental group. As it is illustrated by the image, chicks from the control group tend to stay close to the border of the arena and to move very little, while chicks from experimental groups travel a lot following the robot. To quantify this different attitude to the robot we compared for every experiment distances between the chick group centers and the robot, averaged for the whole experiment. The Kruskal-Wallis test shows that the two datasets are statistically different $\left(p=0.52 \times 10^{-6}\right)$, see also Figure 17 . We can see that chicks from the control never stayed close to the robot.

Thus, we have demonstrated that, first, the mobile robots can be successfully integrated into the animal society by using the imprinting mechanism and that, second, groups composed of chicks that were subject to the imprinting procedure demonstrated the robot following behavior that was never observed in groups composed of chicks that did not undergo the imprinting procedure.

\subsection{Demonstrations}

Here we give several examples of behavioral experiments that we did by using the PoulBot robots. All experiments that we conducted are open field, that is, 

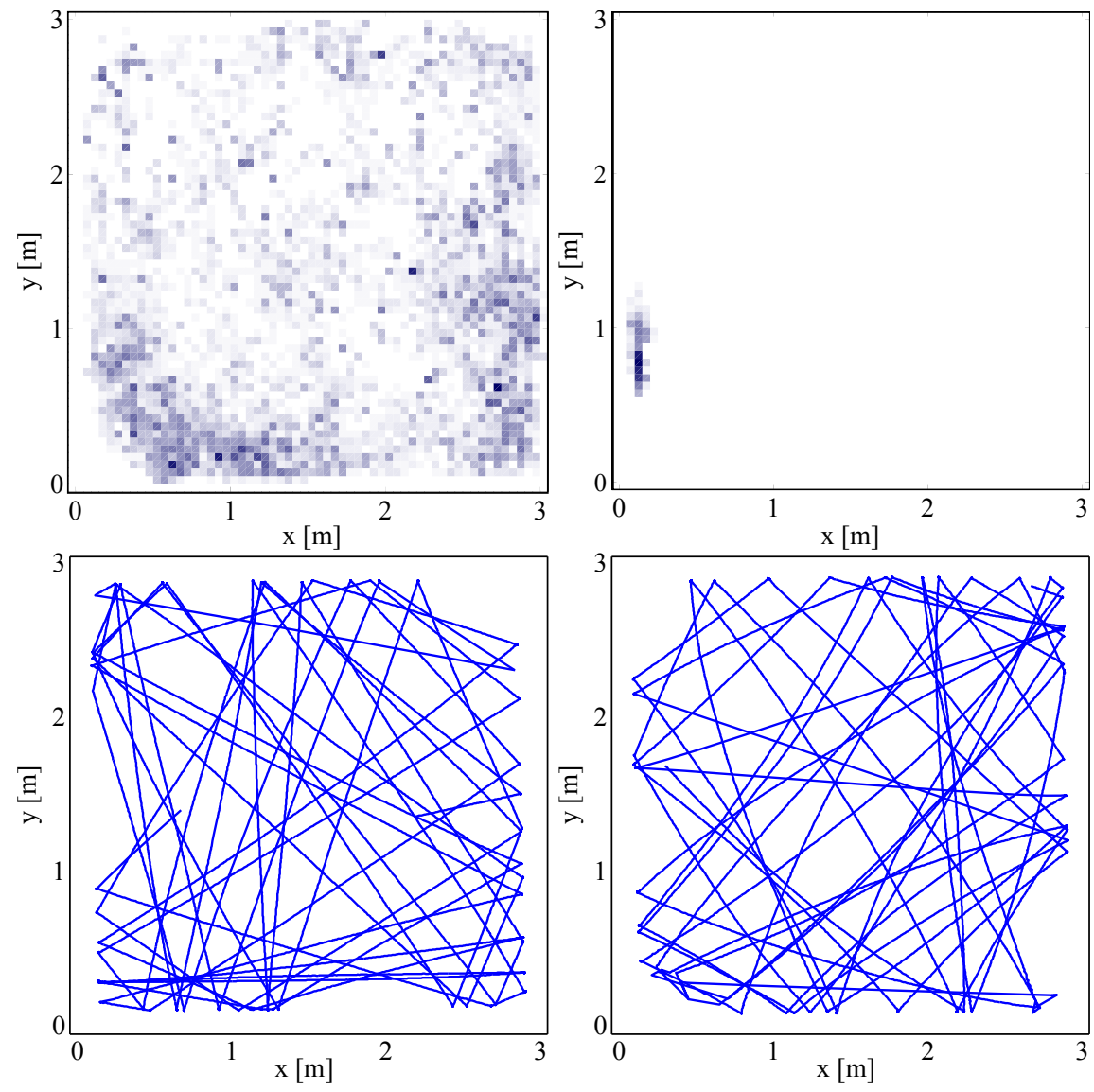

Figure 16: Examples of arena occupancy grids for two groups of chicks: on the top left plot is a test group composed of chicks that underwent the imprinting procedure and on the top right plot is a control group composed of chicks that were never imprinted on the robot. The step of the grid is $50 \mathrm{~mm}$. On the two plots below are the corresponding trajectories of the robot. It can be seen that the chicks from the control group stay immobile unlike the chicks from the test group that travel a lot around the arena. 


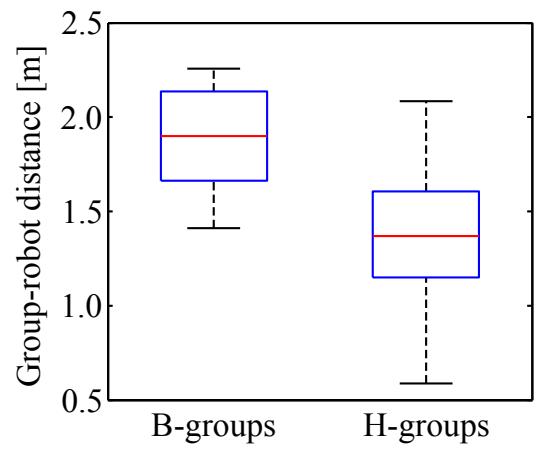

Figure 17: Boxplots corresponding to the four datasets, where the first two are composed of values of mean distances between a chick group and a robot in group following experiments, collected for the control groups ( $B$-groups), composed of the chicks that didn't undergo the imprinting procedure, and for the experimental groups ( $H$-groups), composed of chicks that were subject to the imprinting procedure. For every box, the central mark corresponds to the median value, the top and bottom edges of the box are the 25 th and 75 th percentiles. The whiskers mark two the most extreme data points.

the animals and robots are released on the flat arena, and their behavior is then observed. A number of publications have been prepared based on the collected data and are being submitted to biological journals.

\subsubsection{The following dynamics analysis}

In these experiments, robots and chicks were released together on the arena. The robot emits a calibrated beeping sound and moves at a constant speed; when it reaches a wall of the set-up it turns away with a random angle. We observe the behavior of chicks. This type of experiments was done with individual chicks and in groups. In individual tests we tested the reaction of animals on the presence of the robot and estimated the level of imprinting of every chick, this experiment was usually done in the very beginning of each batch of experiments with the freshly hatched and imprinted chicks and then repeated in the very end of experiments to check how the following behavior changed as a result of ageing and learning. In group experiments we investigated the group dynamics in the robot following, and how individual imprinting level and the social interactions 
between chicks affect it. The main focus in our studies was on this type of experiments.

\subsubsection{The following with feedback experiments}

In these experiments, we investigated how introduction of behavioral feedback from the robot to the group could improve the following dynamics. The task for the robot was to bring chicks from one point of the arena to another. The robot was provided with the positions of chicks and could adapt its behavior by executing one of several strategies: if the chicks stayed behind the robot could wait for them, or it could go to the goal position and back towards the chicks, as if indicating where to go. Alternatively, the robot could come back and pass by the group to pick it up.

\subsubsection{Two-choice experiments}

We did several types of binary choice experiments, the first one is a following experiments with a group of chicks, but here instead of one we introduced two identical robots on the arena and studied how the group selects which one to follow.

In the second type of binary choice we again use two robots equipped but this time with different color patterns. The robots were moving in the opposite parts of the arena. One robot carried the pattern that was familiar to the chicks since it was used during the imprinting procedure, the second had a pattern that was different in color or in shape. Chicks were released in the center of the arena and we observed which robot they selected to join.

\section{Conclusion}

Robotic devices are becoming a common tool in behavioral biology research, thanks to the ability to test in an automatic way the animal reaction to various isolated or multimodal stimuli. However, their complexity, perceptual capabilities, autonomy, and ability are usually very limited. Very few research projects 
work on robots capable of interacting autonomously with animals by using social mechanisms.

The aim that we pursued in this study was to build an autonomous mobile robot that can be accepted by chickens as a part of the group. As a model animal, we selected the domestic chicken. We developed the mobile robot PoulBot - a modular mobile robot that can be expanded with various extension modules if necessary. To do indoor animal-robot experiments, we built an experimental setup composed of an experimental arena with controlled light conditions, a video camera fixed on top of the arena, and a PC responsible for recording the experimental video, running the computer vision system, and providing the robot with the coordinates of detected objects through a Wi-Fi connection. We used the presented robots and monitoring tools to run more than 500 hours of experiments. We have demonstrated that the PoulBot robot can be successfully socially integrated into the animal group, thanks to the imprinting mechanism that is confirmed by the following behavior demonstrated by imprinted chicks.

The designed system opens new opportunities in the study of behavior in domestic fowl by using mobile robots. Being socially integrated into the animal group, mobile robots can profit from the positive feedback mechanism that plays key roles in animal collective behavior. The predictability and repeatability of the robotic behavior guarantees that experiments can be repeated many times in the same conditions; that is not the case when specially trained animals or teleoperated robots are used for the same purpose. In the first case, an animal must be trained for a long time and even in this case we have a less flexible control over its behavior. Moreover, when a real animal is used, we cannot test the reaction of animals to "non-natural" behaviors. In the second case, operators of the robot introduce an inevitable bias into the robotic behavior.

To summarize, in this paper we presented a novel work that achieved social integration of several robots into a group of vertebrates. We believe that our system provides a useful toolset in the study of fundamental social mechanisms and advance our understanding of collective animal behavior. The field of animal-robot interaction is young and we foresee numerous new and promis- 
ing directions of research. One direct field of application is research in animal behavior. A number of monitoring and data analysis tools was recently developed to produce automated quantitative ethograms $[30,58,59]$. By combining embedded social robotic lures with automated ethograms we can achieve an unparalleled automation of ethological experimentation. In the long term, we believe that our work could be a basis for the intelligent systems able to manage domestic and wild life animal pests or resources. Many domesticated animal species are social animals, e.g., poultry, cattle, sheep, goat [60]. The concepts presented here could be applied to such animal societies leading to various agricultural applications such as low-stress management of live-stock. We can also imagine intelligent systems capable of interacting and influencing the behavior of unwanted pests to move them away of specific places [61]. Or, on the other hand, to attract valuable animals used as natural resources, for example, schools of fish. Finally, by designing mixed societies of animal and robots we open the way to hybrid systems, where the artificial agents are enhanced by animal capabilities and, in the opposite way, provide their capabilities to animals. For example, animals have very good perception abilities for sounds, vision, and more importantly smell. Artificial agents presenting similar capabilities are still very far from animal perceptual efficiency. One can also envision animals making use of the artificial agent capabilities such as long range communication that is easily performed by robots. This would lead to novel group behavior based on natural short range and artificial long range perception.

\section{References}

[1] J. Krause, G. Ruxton, Living in Groups, Oxford University Press, 2002.

[2] O. Petit, R. Bon, Decision-making processes: The case of collective movements, Behavioural processes 84 (2010) 635-647.

[3] M.-H. Pillot, J. Gautrais, P. Arrufat, I. D. Couzin, R. Bon, J.-L. Deneubourg, Scalable rules for coherent group motion in a gregarious vertebrate, PLoS ONE 6 (1). 
[4] N. E. Collias, E. C. Collias, Social organization of a red junglefowl, gallus gallus, population related to evolution theory, Animal Behaviour 51 (6) (1996) $1337-1354$.

[5] C. Sueur, O. Petit, J. Deneubourg, I. Couzin, Group size, grooming and social cohesion in primates: a modeling approach based on group structure, Behavioral Ecology and Sociobiology.

[6] M. Pillot, J. Deneubourg, Collective movements, initiation and stops: diversity of situations and law of parsimony, Behavioural Processes 84 (2010) $657-661$.

[7] S. Reebs, Can a minority of informed leaders determine the foraging movements of a fish shoal?, Animal Behaviour 59(2) (2000) 403-409.

[8] J. Krause, A. F. Winfield, J. Deneubourg, Interactive robots in experimental biology, Trends Ecol Evol 26 (7) (2011) 369-375.

[9] J. Halloy, G. Sempo, G. Caprari, C. Rivault, M. Asadpour, F. Tache, I. Said, V. Durier, S. Canonge, J. M. Ame, C. Detrain, N. Correll, A. Martinoli, F. Mondada, R. Siegwart, J.-L. Deneubourg, Social integration of robots into groups of cockroaches to control self-organized choices, Science 318 (5853) (2007) 1155-1158.

[10] D. T. Swain, I. D. Couzin, N. E. Leonard, Real-time feedback-controlled robotic fish for behavioral experiments with fish schools., Proceedings of the IEEE 100 (1) (2012) 150-163.

[11] F. Bonnet, P. Rétornaz, J. Halloy, A. Gribovskiy, F. Mondada, Development of a mobile robot to study the collective behavior of zebrafish, in: IEEE International Conference on Biomedical Robotics and Biomechatronics, 2012.

[12] T. Landgraf, H. Nguyen, S. Forgo, J. Schneider, J. Schrer, C. Krger, H. Matzke, R. Clment, J. Krause, R. Rojas, Interactive robotic fish for the 
analysis of swarm behavior, in: Y. Tan, Y. Shi, H. Mo (Eds.), Advances in Swarm Intelligence, Vol. 7928 of Lecture Notes in Computer Science, Springer Berlin Heidelberg, 2013, pp. 1-10.

[13] A. Goth, C. S. Evans, Social responses without early experience: Australian brush-turkey chicks use visual cues to aggregate with conspecifics, Journal of experimental biology 207 (2004) 2199-208.

[14] E. de Margerie, S. Lumineau, C. Houdelier, M.-A. Richard Yris, Influence of a mobile robot on the spatial behaviour of quail chicks, Bioinspiration \& Biomimetics 6 .

[15] A. Gribovskiy, J. I. Halloy, J.-L. Deneubourg, H. Bleuler, F. Mondada, Towards Mixed Societies of Chickens and Robots, in: Proceedings of the IEEE/RSJ 2010 International Conference on Intelligent Robots and Systems (IROS 2010), 2010.

[16] A. Gribovskiy, F. Mondada, Real-time audio-visual calls detection system for a chicken robot, in: International Conference on Advanced Robotics, 2009, 2009.

[17] A. Gribovskiy, J. Halloy, J.-L. Deneubourg, F. Mondada, Building a safe robot for behavioral biology experiments, in: IEEE International Conference on Robotics and Biomimetics (ROBIO 2012), 2012.

[18] E. H. Hess, Imprinting: Early Experience in the Developmental Psychology, Van Nostrand Reinhold, NY, 1973.

[19] D. McFarland, Animal Behaviour: Psychobiology, Ethology and Evolution, Longman, 1999.

[20] N. E. Collias, M. Joos, The spectrographic analysis of sound signals of the domestic fowl, Behaviour 5 (3) (1953) pp. 175-188.

[21] N. E. Collias, The vocal repertoire of the red junglefowl: A spectrographic classification and the code of communication, The Condor 89 (3) (1987) pp. $510-524$. 
[22] E. Clara, L. Regolin, M. Zanforlin, G. Vallortigara, Domestic chicks perceive stereokinetic illusions, Perception 35(7) (2006) 983-92.

[23] E. Mascalzoni, L. Regolin, G. Vallortigara, Innate sensitivity for selfpropelled causal agency in newly hatched chicks, Proceedings of the National Academy of Sciences 107(9) (2010) 44834485.

[24] T. Lochmatter, P. Roduit, C. Cianci, N. Correll, J. Jacot, A. Martinoli, SwisTrack - a flexible open source tracking software for multi-agent systems, in: IROS, 2008, pp. 4004-4010.

[25] G. Bradski, The OpenCV Library, Dr. Dobb's Journal of Software Tools.

[26] N. Nasios, A. Bors, Variational learning for gaussian mixture models, Systems, Man, and Cybernetics, Part B: Cybernetics, IEEE Transactions on 36 (4) (2006) $849-862$.

[27] C. M. Bishop, Pattern Recognition and Machine Learning, Springer, 2006.

[28] J.-M. Valin, F. Michaud, J. Rouat, Robust localization and tracking of simultaneous moving sound sources using beamforming and particle filtering, Robotics and Autonomous Systems 55 (3) (2007) 216-228.

[29] M. Isard, A. Blake, Condensation - conditional density propagation for visual tracking, International Journal of Computer Vision 29 (1998) 5-28.

[30] K. Branson, A. A. Robie, J. Bender, P. Perona, M. H. Dickinson, Highthroughput ethomics in large groups of drosophila, Nat Meth 6 (6) (2009) $451-457$.

[31] R. Tsai, A versatile camera calibration technique for high-accuracy $3 \mathrm{~d}$ machine vision metrology using off-the-shelf tv cameras and lenses, Robotics and Automation, IEEE Journal of 3 (4) (1987) $323-344$.

[32] F. Mondada, J. Halloy, A. Martinoli, N. Correll, A. Gribovskiy, G. Sempo, R. Siegwart, J. Deneubourg, Handbook of Collective Robotics: Fundamen- 
tals and Challenges, Pan Stanford, 2012, Ch. A general methodology for the control of mixed natural-artificial societies, to appear.

[33] P. H. Klopfer, Imprinting: A reassessment, Science 156 (1967) 1394-1396.

[34] P. P. G. Bateson, J. B. Jaeckel, Imprinting: Correlations between activities of chicks during training and testing, Animal Behaviour 22 (1974) 899-906.

[35] A. Ham, D. Osorio, Colour preferences and colour vision in poultry chicks, Proc Biol Sci. 274 (2007) 19411948.

[36] H. S. Van Kampen, J. J. Bolhuis, Auditory learning and filial imprinting in the chick, Behaviour 117 (1991) 303-319.

[37] M. Bonani, V. Longchamp, S. Magnenat, P. Rétornaz, D. Burnier, G. Roulet, H. Bleuler, F. Mondada, The marXbot, a miniature mobile robot opening new perspectives for the collective-robotic research, in: 2010 IEEE/RSJ International Conference on Intelligent Robots and Systems, 2010.

[38] BOSCH, Can specification, Tech. rep., BOSCH (1991).

[39] S. Magnenat, P. Rétornaz, M. Bonani, V. Longchamp, F. Mondada, Aseba: A modular architecture for event-based control of complex robots, IEEE/ASME Transactions on Mechatronics 16 (2011) 321 - 329.

[40] F. Maekawa, O. Komine, K. Sato, T. Kanamatsu, M. Uchimura, K. Tanaka, H. Ohki-Hamazaki, Imprinting modulates processing of visual information in the visual wulst of chicks, BMC Neuroscience 7 (1) (2006) 75.

[41] P. S. Naidu, Sensor Array Signal Processing, CRC Press, Inc., 2000.

[42] D. H. Johnson, D. E. Dugeon, Array Signal Processing: Concepts and Techniques, Prentice Hall, 1993.

[43] J. Christensen, J. Hald, Beamforming, Tech. rep., Bruel\&Kjaer (2004). 
[44] D. T. Blumstein, D. J. Mennill, P. Clemins, L. Girod, K. Yao, G. Patricelli, J. L. Deppe, A. H. Krakauer, C. Clark, K. A. Cortopassi, S. F. Hanser, B. McCowan, A. M. Ali, A. N. G. Kirschel, Acoustic monitoring in terrestrial environments using microphone arrays: applications, technological considerations and prospectus, Journal of Applied Ecology 48 (3) (2011) 758-767.

[45] H. Okuno, K. Nakadai, H.-D. Kim, Robot audition: Missing feature theory approach and active audition, in: C. Pradalier, R. Siegwart, G. Hirzinger (Eds.), Robotics Research, Vol. 70 of Springer Tracts in Advanced Robotics, Springer Berlin / Heidelberg, 2011, pp. 227-244.

[46] S. Brière, J. M. Valin, F. Michaud, D. Letourneau, Embedded auditory system for small mobile robots, in: IEEE International Conference on Robotics and Automation, 2008, pp. 3463-3468.

[47] Y. Sasaki, S. Thompson, M. Kaneyoshi, S. Kagami, Map-generation and identification of multiple sound sources from robot in motion, in: F. Asano (Ed.), International Conference on Intelligent Robots and Systems (IROS 2010), Vol. 3, 2010, pp. 2404-2410.

[48] Y. Tamai, Y. Sasaki, S. Kagami, H. Mizoguchi, Three ring microphone array for $3 \mathrm{~d}$ sound localization and separation for mobile robot audition, in: IEEE/RSJ International Conference on Intelligent Robots and Systems, 2005, pp. $4172-4177$.

[49] M. S. Brandstein, D. B. Ward (Eds.), Microphone Arrays: Signal Processing Techniques and Applications, Springer-Verlag, 2001.

[50] G. DeMuth, Frequency domain beamforming techniques, in: IEEE International Conference on Acoustics, Speech, and Signal Processing, Vol. 2, 1977, pp. 713-715.

[51] N. Tashev, Gain self-calibration procedure for microphone arrays, IEEE 
International Conference on Multimedia and Expo (ICME 2004) 2 (2004) 983-986.

[52] M. J. Matarić, M. J. Michaud, Springer Handbook of Robotics, Springer, 2008, Ch. Behavior-Based Systems, pp. 891-909.

[53] R. C. Arkin, Behavior-Based Robotics, The MIT Press, 1998.

[54] M. Zinn, O. Khatib, B. Roth, J. Salisbury, Playing it safe [human-friendly robots], Robotics Automation Magazine, IEEE 11 (2) (2004) 12 - 21.

[55] I. Ulrich, J. Borenstein, VFH+: reliable obstacle avoidance for fast mobile robots, in: ICRA, 1998.

[56] S. Roberts, Novelty detection using extreme value statistics, IEE Proceedings Vision, Image and Signal Processing 146 (3) (1999) 124 -129.

[57] A. O. Ramsay, E. H. Hess, A laboratory approach to the study of imprinting, The Wilson Bulletin 66 (3) (1954) pp. 196-206.

[58] H. Dankert, L. Wang, E. D. Hoopfer, D. J. Anderson, P. Perona, Automated monitoring and analysis of social behavior in drosophila, Nat Meth 6 (4) (2009) 297-303.

[59] M. Reiser, The ethomics era?, Nat Meth 6 (6) (2009) 413-414.

[60] L. J. Keeling, H. Gonyou, Social behavior in farm animals, CABI Publishing, Wallingford UK, 2001.

[61] J. Tracey, M. Bomford, Q. Hart, G. Saunders, R. Sinclair, Managing Bird Damage to Fruit and Other Horticultural Crops, Bureau of Rural Sciences, Canberra, 2007. 